$7-1-2003$

\title{
Entropic Characterization of Distributive Mixing in Polymer Processing Equipment
}

\author{
Winston Wang \\ Case Western Reserve University, wxw6@po.cwru.edu \\ Ica Manas Zloczower \\ Case Western Reserve University, ica.manas-zloczower@case.edu \\ Miron Kaufman \\ Cleveland State University, m.kaufman@csuohio.edu
}

Follow this and additional works at: https://engagedscholarship.csuohio.edu/sciphysics_facpub

Part of the Physics Commons

How does access to this work benefit you? Let us know!

Publisher's Statement

This is the accepted version of the following article: Winston Wang, Ica Manas-Zloczower and Miron Kaufman, "Entropic characterization of distributive mixing in polymer processing equipment," AIChE J. 49 (7), 1637-1644 (2003), which has been published in final form at http://onlinelibrary.wiley.com/doi/10.1002/aic.690490704/pdf

\section{Repository Citation}

Wang, Winston; Zloczower, Ica Manas; and Kaufman, Miron, "Entropic Characterization of Distributive Mixing in Polymer Processing Equipment" (2003). Physics Faculty Publications. 235.

https://engagedscholarship.csuohio.edu/sciphysics_facpub/235

This Article is brought to you for free and open access by the Physics Department at EngagedScholarship@CSU. It has been accepted for inclusion in Physics Faculty Publications by an authorized administrator of EngagedScholarship@CSU. For more information, please contact library.es@csuohio.edu. 


\title{
Entropic Characterization of Distributive Mixing in Polymer Processing Equipment
}

\author{
Winston Wang and Ica Manas-Zloczower
}

\author{
Miron Kaufman
}

Mixing is an integral component of most polymer processing operations as material properties are highly influenced by the quality of mixing. The degree of distributive mixing (system homogeneity) is assessed by calculating the evolution of Renyi relative entropies for the minor component along a continuous processing equipment. The Renyi entropy involves a $\beta$ parameter, which represents weighting given to the concentration of the minor component in small, localized regions. Different aspects of mixing can thus be analyzed, from the amount of void spaces to the concentration of the region where mixing is the worst in terms of the minor component. This method provides a unified, rigorous and flexible way of characterizing distributive mixing. Specifically, distributive mixing is analyzed in a numerical simulation of a twin-flight single screw extruder by using particle tracking as a method of describing the mixing process dynamics. Renyi entropies are used to examine three different processing conditions in a twin-flight, single screw extruder. By changing the throttle ratio, the optimal extruder length varied. The relationship between the optimal length and average residence time was good.

\section{Introduction}

Entropy is a rigorous measure of disorder or system homogeneity. Various entropic measures are used in many fields of science. In statistical mechanics the Boltzmann thermodynamic entropy is determined by the partition of the phase space of positions and momenta and measures the disorder or lack of information about a physical system. Shannon (1948) developed the theory of information by using the entropy to measure the amount of information transmitted through electronic channels. Brooks (1988) used informational entropy to model biological evolution. In the field of cognitive science, informational entropy is used to model memory (Allen et al., 1998). Georgescu-Roegen (1987) used entropy to model economic processes. Grassberger and Procaccia (1984) apply entropy to model dynamic systems. Renyi (1973) generalized the concept of entropy to measure different aspects of system homogeneity. In the medical field
(Ivanov et al., 1999; Kantz and Schreiber, 1995; Kurths et al., 1995), Renyi entropies are used to characterize the time series of heartbeats.

The goal of the present work is to use Renyi entropies for defining new distributive mixing criteria for the purpose of evaluating polymer processing equipment. These new criteria will help to evaluate and assist in the improvement of current and future compounding machines.

In distributive mixing, repeated rearrangement of the minor component enhances system homogeneity. In continuous mixing processes, composition uniformity at the emerging stream is directly related to the material residence time distribution.

To facilitate a quantitative measure of distributive mixing, Danckwerts (1953) proposed to examine the intensity of segregation and the scale of segregation.

The intensity of segregation reflects how the composition of the mixture in the local area deviates from the average composition of the whole mixture. The lower the intensity of 
segregation is, the less deviation there is among the local areas, resulting in a better mixture.

The scale of segregation represents the sizes of local domains of each component. It is a useful measure of small-scale texture, where smaller domains indicate better mixing.

Lately, there has been a great deal of interest in the development of mixing criteria for polymer processing equipment. A review of the approaches taken by various research groups follows.

Ottino (1989) and his group have done extensive work in understanding and characterization of mixing. To assess distributive mixing in chaotic flows, he introduced the use of the length stretch of the interface between two immiscible viscous fluids (Chakravarthy and Ottino, 1996).

Yao (Yao et al., 1996) used a local mixing efficiency criteria based on the specific rate of stretching of interfacial area to analyze distributive mixing in a pin mixing section for single screw extruders. Their results are limited to a 2-D finite difference analysis. Lawal and Kalyon (1995) simulated the isothermal flow in single and co-rotating twin screw extruders and used various tools of dynamics to quantify distributive mixing. Kwon proposed using "deformation characteristics" based on the Green deformation tensor as a strain and implicit mixing measure of screw extrusion processes (Kwon et al., 1994).

Passive tracers have often been used to help evaluate mixing in a variety of equipment. These passive tracers are assumed to not affect the flow field and not interact with each other. Wong and Manas-Zloczower (1994) studied distributive mixing in internal mixers by tracking the motion of passive tracers. Distributive mixing was quantified in terms of the probability density function of a pairwise correlation function. Avalosse (1996) used a similar technique to study mixing in a stirred tank. Mackley and Saraiva (1999) used kinematic mixing rates and concentration distributions of passive tracers to look at mixing in the oscillatory flow within baffled tubes.

Meijer and coworkers used the mapping method to analyze mixing behavior in different geometries. The flow is divided into a large number of subdomains whose boundaries are tracked for a period of time or space. During tracking, various quantities relevant for distributive mixing, such as concentration, residence time, or morphology can be computed (Anderson et al., 1999; Galaktionov et al., 2001).

Yoshingaga et al. (2000) numerically simulated distributive mixing in a twin screw extruder using residence time distributions and distribution of length stretch between tracers. $\mathrm{Li}$ and Manas-Zloczower (1995a,b) and Cheng and ManasZloczower (1998) have also used tracers to study the dynamics of the mixing process and proposed several criteria to quantify distributive mixing. Shearer and Tzoganakis (1999) used reactive polymer tracers as a microscopic probe of the interfacial surface area between two polymer melts and found a nonlinear relationship between screw speed and mixing performance.

Residence time distributions have been studied in conjunction with their use in evaluating distributive mixing in processing equipment. Bigio and coworkers modeled and analyzed the mean residence time and residence time distribution in a twin screw extruder. They found a correlation between percent drag flow and residence time (Gao et al., 2000;
Gasner et al., 1999). That is, a larger percentage of drag flow meant the fraction of residence time in the mixing elements decreased, resulting in poorer mixing quality.

The variety of approaches to characterize mixing is symptomatic of the complexity of the task. In fact Ottino (1989) has stated that a distributive mixing index must be selected according to the specific application, and it must be tailored to the particular fluid mechanics problem.

In this work we explore the idea of using Renyi entropies to measure the degree of distributive mixing in polymeric systems. A well-distributed multicomponent system will have high entropy. Renyi entropies allow examination of different aspects of system homogeneity. For example, one Renyi entropy allows the quantification of void spaces (regions with no minor component present), another one focuses on the region with greatest intensity, while others look at the overall level of mixing in the system.

By using the appropriate Renyi entropy, we can focus on different aspects of distributive mixing which can be obtained. In addition this method is independent of the geometry of the system, which allows it to be applied universally to any type of mixing equipment. Also, although in this work we will restrict the analysis to a two-component system, Renyi entropies can be readily generalized to systems with more than two components. So the flexibility and versatility of Renyi entropies provide a unified approach for characterizing different aspects of distributive mixing.

\section{Renyi Entropies}

The information entropy for a particular experimental condition with a set of $M$ possible outcomes is

$$
S=-\sum_{j=1}^{M} p_{j} \ln p_{j}
$$

where $p_{j}$ is the relative frequency of outcome No. $j$. Equation 1 is the standard formulation (Shannon, 1948) of uncertainty as it satisfies certain common-sense requirements: (i) the lowest entropy ( $S=0$ ) corresponds to one of the $p$ 's being 1 and the rest being zero (that is, total information, perfect order); (ii) the largest value for the entropy $S=\ln (M)$ is achieved when all $p$ 's are equal to each other (that is, the absence of any information, complete disorder); and (iii) $S$ is additive over partitions of the outcomes. If the last axiom is relaxed to consider only statistically independent partitions, Renyi (1973) determined that the information entropy can be replaced with a one-variable function

$$
S(\beta)=\frac{\ln \left(\sum_{j=1}^{M} p_{j}^{\beta}\right)}{1-\beta}
$$

In the limit $\beta \rightarrow 1$, Eq. 2 reduces to Eq. 1 . If $\beta>0$, the function defined in Eq. 2 is maximized when all $p$ 's are equal to each other, while, if $\beta<0$, the function in Eq. 2 is minimized when all the $p$ 's are equal thus violating axiom ii above. For this reason, we consider in our study only nonnegative values of the parameter $\beta$. It follows that: $0 \leq S(\beta) \leq \ln (M)$. Thus, 
$S(\beta) / \ln (M)$ constitutes an index of homogeneity: it is 1 for total disorder or homogeneity and is small for high order or segregation. Shannon called this ratio the relative entropy. The relative entropy subtracted from unity constitutes a measure of the deviation of the concentration in each local area from the ideal distribution. Thus, it is equivalent to a measure of intensity of segregation.

Renyi dimensions are used to characterize multifractals (fractal structure with a probability distribution defined on it) (Bunde et al., 1990). The relative entropy has the following geometric interpretation. If we cover the available space with $M$ equal size boxes, than $M=\epsilon^{-D}$, where $\epsilon$ is the ratio of the linear size of the box to the linear size of the system and $D$ is the dimension of the embedding space. $\epsilon$ gives a measure of the system scale of segregation. As we increase the number of boxes $M$, or decrease the scaling factor $\epsilon$, we expect that $S(\beta) \approx-d(\beta) \ln (\epsilon)$ where $d(\beta)$ is the Renyi generalized dimension. Thus, the relative entropy is equal to the ratio of the generalized dimension to the embedding space dimension

$$
S_{\text {relative }}(\beta)=S(\beta) / \ln (M)=d(\beta) / D
$$

The interpretation of $d(\beta)$ as a dimension originates in the case $\beta \rightarrow 0$. According to Eq. $2, S(0)=\ln$ (number of occupied boxes). In this case equal weight is given to all boxes containing particles, independent of the number of particles present in the box. Since the fractal (Hausdorff) dimension is defined as

$$
\begin{array}{r}
d_{\text {Hausdorff }}=\ln (\text { number of occupied boxes }) /-\ln (\epsilon) \\
=S(0) /-\ln (\epsilon)
\end{array}
$$

The entropy in the limit $\beta \rightarrow 0$ is determined by the distribution of empty bins or void spaces.

For $\beta=1$, one gets the information dimension as $S(1)$ is the Shannon entropy, which measures the lack of information. The more random a data set is, the less information it can convey. Therefore, the higher the information dimension is, the more random the system is. Consequently, it is better distributed. For $\beta=2$, one gets the correlation dimension as $S(2)$ is related to the logarithm of the correlation sum. As $M \rightarrow \propto$, the result approaches the correlation sum function. Taking the slope of a linear fit of $S(2)$ vs. $\ln (M)$ for various number of bins $M$, we can calculate the correlation dimension in a range of resolutions related to the system scale of segregation. The correlation dimension is most appropriate for discrete particle systems. As $\beta \rightarrow \alpha$, the sum in Eq. 2 is dominated by the largest probability $p_{\max }$

$$
S(\infty) \approx-\ln \left(p_{\max }\right) \text { and } d(\infty) \approx \ln \left(p_{\max }\right) / \ln (\epsilon)
$$

This entropy is useful when it is necessary to evaluate mixing based on the regions of largest concentration/poorest distributive mixing in the system.

In this work we test the use of the relative entropy $S_{\text {relative }}(\beta)=d(\beta) / D$ as an index of homogenization (opposite of intensity of segregation) for two-dimensional (2-D) sections $(D=2)$ along an extruder.

\section{Results and Discussion}

Renyi entropies can be used for distributive mixing characterization in any polymer processing equipment. For the purpose of illustrating the methodology, we examine a single screw extruder. It should be noted that while single screw extruders are not known for their mixing capabilities, but rather their melting and pumping capabilities, it is still important to understand the degree of mixing which occurs in such a machine.

As described in previous work, finite element simulations for the flow field of a Newtonian fluid in a twin-flight single screw extruder were performed using FIDAP, on a Cray T-94 at the Ohio Supercomputer Center (Wang and Manas-Zloc-

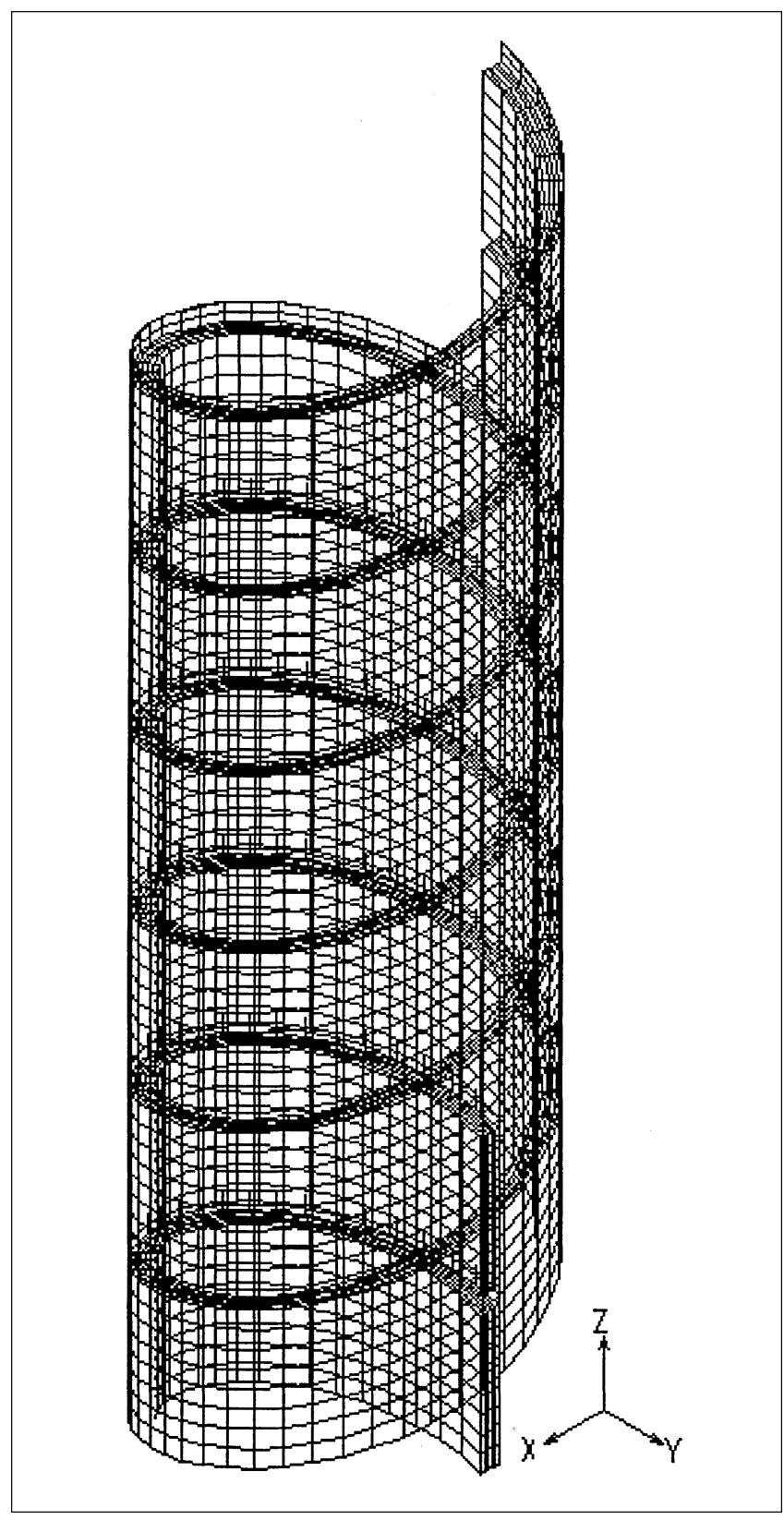

Figure 1. Finite-element mesh for a twin-flight single screw extruder. 
zower, 2001; Wang et al., 2001). Figure 1 shows the finite-element mesh for a three-pitch screw. A pitch is the axial distance required for one helical revolution of the flight. The finite-element mesh in Figure 1 corresponds to a squared pitched extruder (dimensionless length units). Since our system has rotational symmetry, a rotational frame of reference is used so that a single flow field calculation can be used for the full rotation cycle. In the following, unless otherwise specified, we present results obtained using operating conditions of 1 revolution per unit time and a throttle ratio (the ratio of pressure flow to drag flow) $Q p / Q d=+0.5$. From the flow field calculations, we study the mixing process by tracking the motion of fluid elements in the mixing region.

Since the flow field is deterministic, the locations of the fluid elements can be found by integrating the velocity vectors with respect to time

$$
\underline{X}\left(t_{1}\right)=\underline{X}\left(t_{0}\right)+\int_{t_{0}}^{t_{1}} \underline{V}(t) d t
$$

where $\underline{X}\left(t_{1}\right)$ is the location of the fluid element at any time $t_{1}, \underline{X}\left(t_{0}\right)$ is the location of the same fluid element at $t_{0}$, and $\underline{V}(t)$ is the corresponding Lagrangian velocity corresponding to the fluid element. A periodic boundary condition is used to simulate a variable length extruder. Since past works (Cheng and Manas-Zloczower, 1997; Lee and Kwon, 1999) have shown that single screw extruders may exhibit chaotic features of flow, we examined the validity of using a periodic boundary for our fluid tracers. It was determined that the periodic boundary conditions allowed significant savings in computational resources with minimal impact on the traces.

The results from the flow field simulations were used to track the motion of fluid elements in the twin flight single screw extruder. In this project, 5,770 tracers were grouped into 10 clusters of 577 particles each and were evenly distributed in the center region of the entrance cross section of the model extruder. These tracers were followed for different time units with a time step of 0.0001 .

We assume that we continuously inject particles at their initial positions, and that the cross sections are taken from a system at steady state. Doing so allows for the distributive mixing analysis to be done in 2-D axial cross sections independent of time.

To calculate the Renyi Entropies for each spatial distribution, it is necessary to divide up the analysis space, a 2-D axial cross section of the extruder, into bins of equal size/area. The smaller the bins, the higher the resolution of the analysis performed. However, since we estimate probabilities by using frequencies, the number of particles $N$ must be much larger than the number of bins $N \gg M \gg 1$. In addition we have attempted to keep the aspect ratio of the bins as close as possible to $1: 1$.

In the case of the single screw extruder the majority of the results presented here used 360 bins in the theta direction and 8 bins in the radial direction for a total of 2,880 bins. The partitions in the radial direction were calculated so that each and every bin has the same area, which renders the $\Delta r$ closer to the screw larger than the $\Delta r$ near the barrel. It is important to note that some of the bins can be partially or completely covered by the flights. This is necessary in order to simplify the construction of the bins and the calculation of

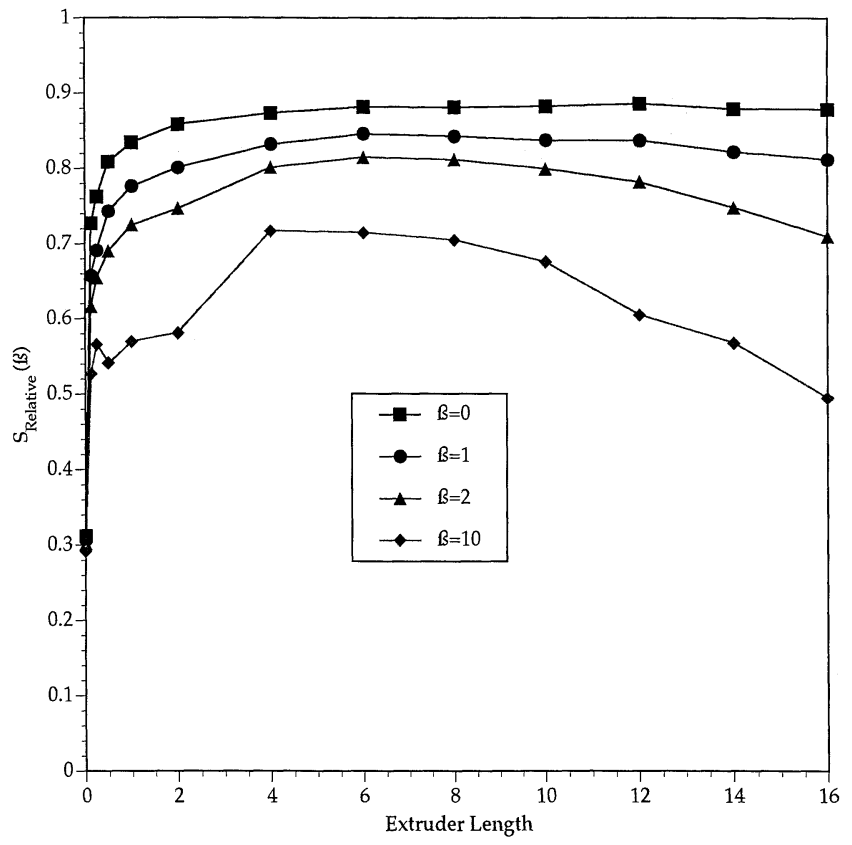

Figure 2. Evolution of $S_{\text {Relaive }}(\beta)$ for $\beta=0,1,2$, and 10 along the axial length of the extruder (No. of pitches) for 5,770 particles and 2,880 bins.

the concentration of particles in each bin. Since the flights are included in the mapped region, the entropies cannot reach maximum value even in the case of perfect mixing/distribution of the tracers (minor component).

Figure 2 shows the evolution of relative Renyi entropies for specific $\beta$ values along the axial length of the extruder. For all $\beta$ values, the relative Renyi entropy reaches a maximum for a six-pitch extruder. Longer extruders show evidence of lower mixing quality.

For $\beta=0$, the relative Renyi entropy rises for the first six pitches, becoming steady for the longer extruders. This indicates that the distribution of void spaces (regions void of minor component) in the system does not change significantly once the extruder exceeds six pitches in length.

The relative Renyi entropy for $\beta=1$ also increases for the first six pitches, but then levels out. This indicates that the intensity of segregation is not reduced appreciably after a six-pitch extruder.

For $\beta=2$, the relative Renyi entropy shows a large improvement over the first six pitches, followed by a modest degradation through the sixteenth pitch.

The relative Renyi entropy corresponding to $\beta=10$ shows an increase through the first 4-6 pitches and then a decrease for longer extruders. As the value of beta increases to 10 , bins with lower concentrations contribute less to the entropy by comparison with the bins of high particle concentrations. So, we see that, for extruders longer than six pitches, demixing is causing the regions of greatest intensity to become even more intense, resulting in poorer mixing. This demixing phenomenon is a consequence of possible chaotic features of flow, which will be examined in a future study.

One can draw an analogy between the information provided by the relative Renyi entropy for $\beta=2$ and the more traditional scale of segregation of the system. Changing the 


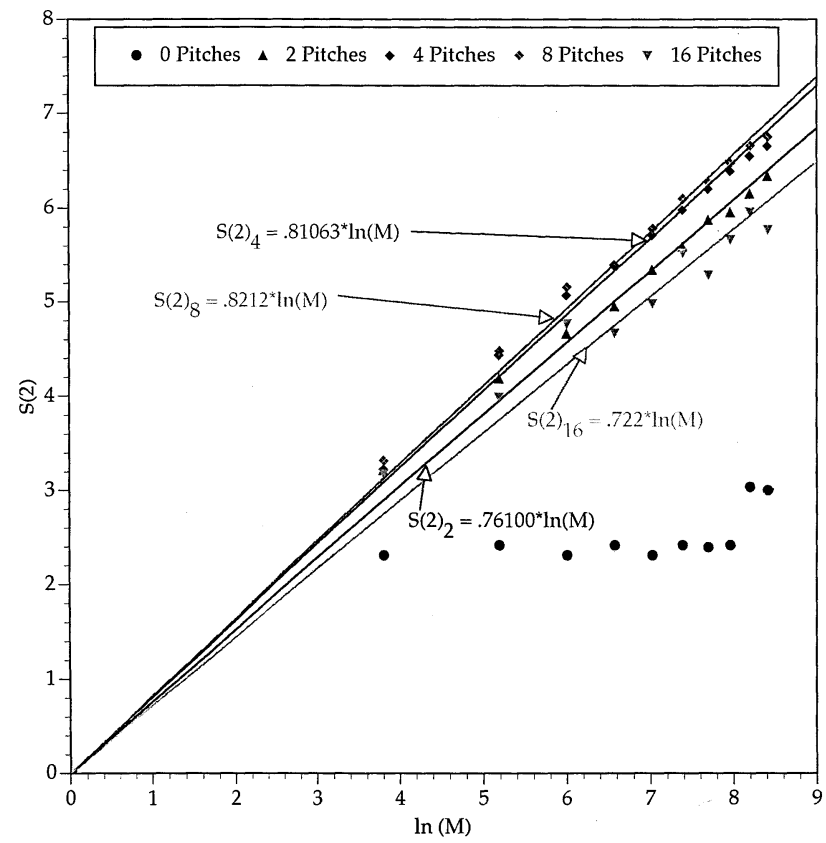

Figure 3. Calculation of relative Renyi entropy for $\boldsymbol{\beta}=\mathbf{2}$ for a range of bin sizes from the linear fit of $S(2)$ vs. $\ln (M)$.

bin size/number of bins in the system, we analyze the degree of distributive mixing at various range orders, spanning from short (large number of bins of small size) to long (small number of bins of large size). Figure 3 plots linear fits of $S(2)$ (the Renyi entropy for $\beta=2$ ) vs. $\ln M$ (number of bins) at different extruder lengths. Since $S(\beta)=0$ for $M=1$, we force the linear fit to pass through zero. One can observe that there is a good linear fit of the data. We note that the data for the entrance cross section fall on a horizontal line simply because, even when using a larger number of bins, the bin size is still larger than the cluster size. Thus, a change in length scale will not reveal a different spatial structure of the mixture.

In Figure 4 we plot the evolution of the correlation dimension calculated via Renyi entropies for a range of bins (that is, the slopes of the lines in Figure 3) along the extruder length. Also plotted in Figure 4 is the relative Renyi entropy for $\beta=2, S_{\text {relative }}(2)$ for a fixed number of bins $(2,880)$ from Figure 2. One can observe that the relative Renyi entropy calculated using a fixed number of bins $(2,880)$ matches well the correlation dimension obtained by spanning a whole range of measures for the scale of segregation, from short $(M=$ $4,500)$ to long range order $(M=45)$. This justifies our choice of 2,880 bins for the results shown, for instance, in Figure 2.

A traditional method of determining distributive mixing is the correlation dimension calculated using the correlation sum (Grassberger and Procaccia, 1983). Correlation sum counts the number of pairs of particles that have a Euclidean distance less than $r$, divided by the total number of pairs of particles $N^{2}$. The correlation sum is calculated for range of $r$ 's $0<r<r_{\max }$ for each spatial distribution. The correlation dimension for the axial cross section is the slope of the regression line of $\ln C(r)$ vs. $\ln (r)$. The evolution of the corre-

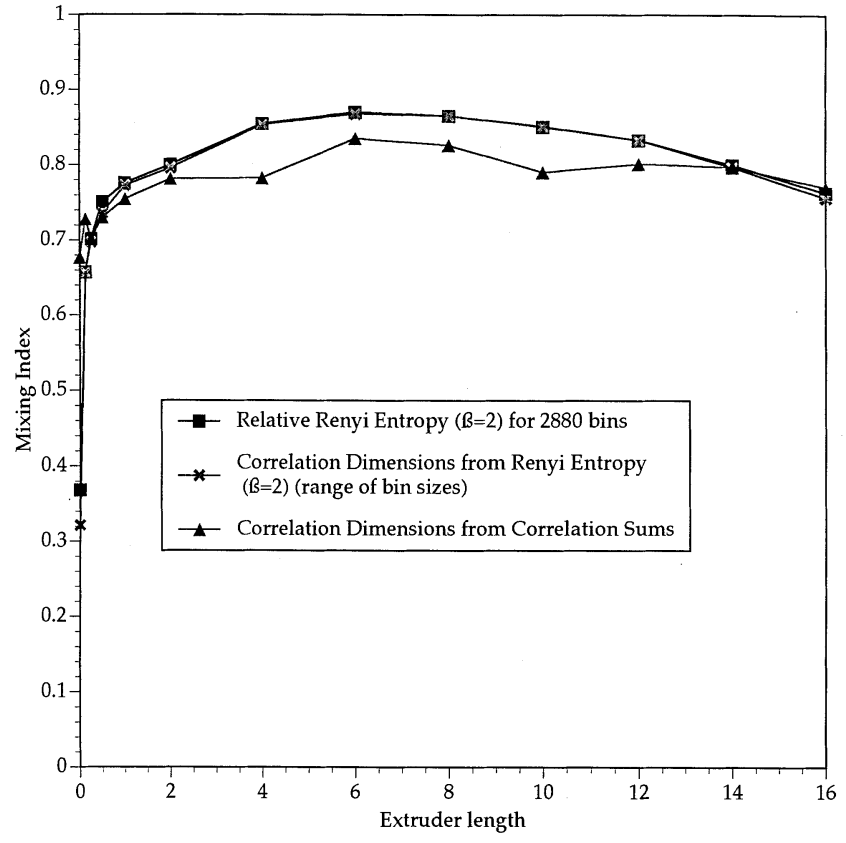

Figure 4. Comparison of the correlation dimension as calculated via correlation sums, via the Renyi entropy $S(2)$ for 2,880 bins, and via the Renyi entropy $S(2)$ for a range of bins.

Each is graphed vs. the axial length of the extruder (No. of pitches).

lation dimension calculated via correlation sum along the extruder length is also shown in Figure 4. The evolution of the correlation dimension from Renyi entropy looks very similar to the one calculated using the more traditional method of the correlation sum.

The Renyi entropy has two major advantages over the more traditional method. First, the Renyi entropies, probing only the concentration within each bin and not the location of the bins, are independent of the geometric scale. This renders the Renyi entropies more universal for distributive mixing characterization. It should be noted also that calculations based on Renyi entropies are on the order of $M$ (number of bins) $* N$ (number of particles), whereas calculations based on correlation sums are on the order of $N^{2}$. Since, as stated previously, $M \ll N$, the computational time is shorter when we use the entropic measures of mixing.

We ascertain next the influence of the number of particles on our entropy estimates. In addition to our original 5,770 particles (10 clusters of 577 particles each), we have looked at the minor component spatial distributions and Renyi entropies using 655 particles arranged in five groups of 131 particles, and 10,940 particles initially arranged in 20 groups of 547 particles each. Figure 5 shows the evolution along the extruder length of the relative Renyi entropy of $\beta=2$ for these three groups. The results look qualitatively similar. The group of 655 particles showed similar trends as the 5,770 particles, but with more scatter. There is no significant improvement by using 10,940 particles when compared with 5,770 particles when looking at the Renyi entropy evolution along the extruder length. 


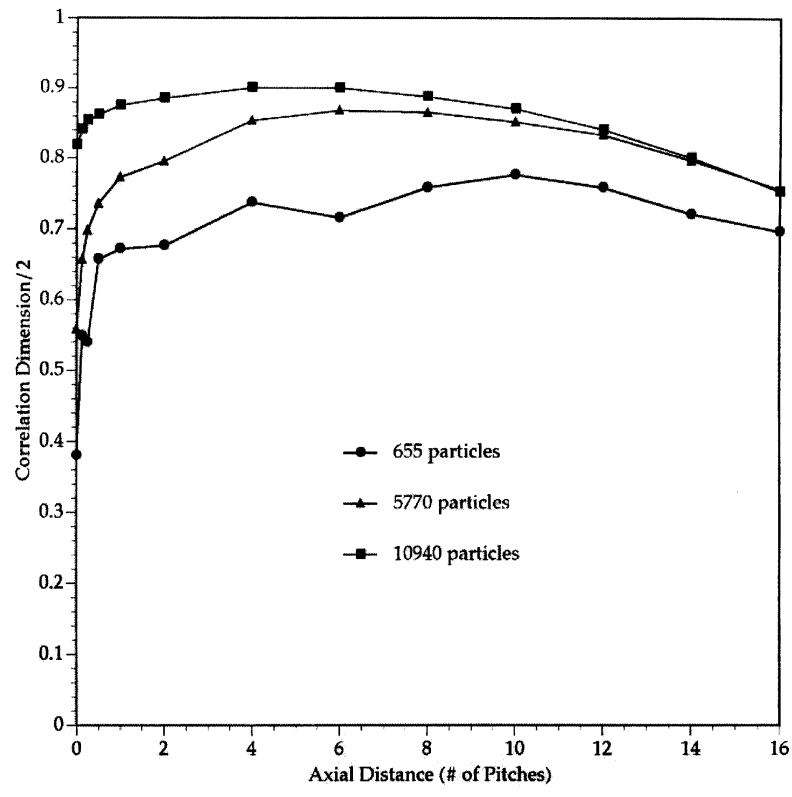

Figure 5. Evolution along the extruder of the correlation dimension from the Renyi entropy of $\beta=2$, for $655,5,770$, and 10,940 particles using 405 , 2,880 , and 6,480 bins, respectively.

To examine the influence of tracer initial positions on distributive mixing, we place the particles in specific regions of the entrance cross section, more specifically near the barrel wall, or near the screw root. For these simulations, we place 4,000 particles in each of the locations and track them for 25 revolutions. The initial locations of the particles are illustrated in Figure 6. Because of symmetry, only half of the cross section is shown. Particles are continuously injected into the system at their initial positions, and the spatial distributions obtained at the exit of the 3rd pitch are at steady state.

The relative Renyi entropies are used to analyze the spatial distributions of the particles from each zone. Figure 7 shows the relative Renyi entropies for Renyi parameter values from zero to ten for particles placed in different initial locations. Particles starting near the screw exhibit the best distributive mixing. These are the particles that move slower and are in a region that has the greatest circulatory space.

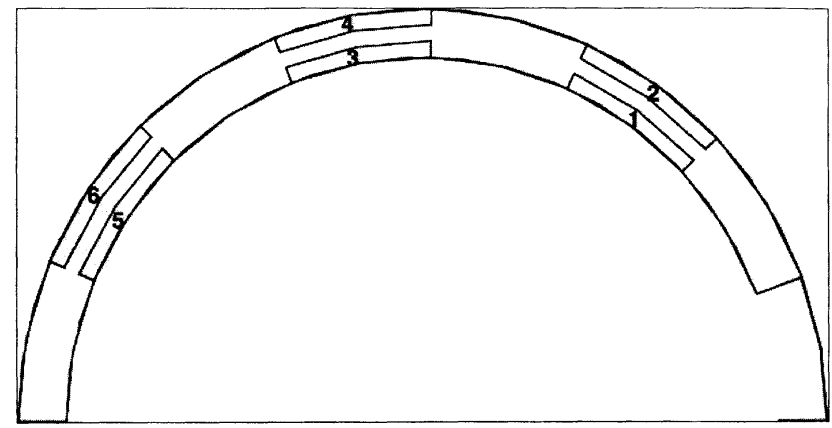

Figure 6. Location of the initial zones used for particle tracking.

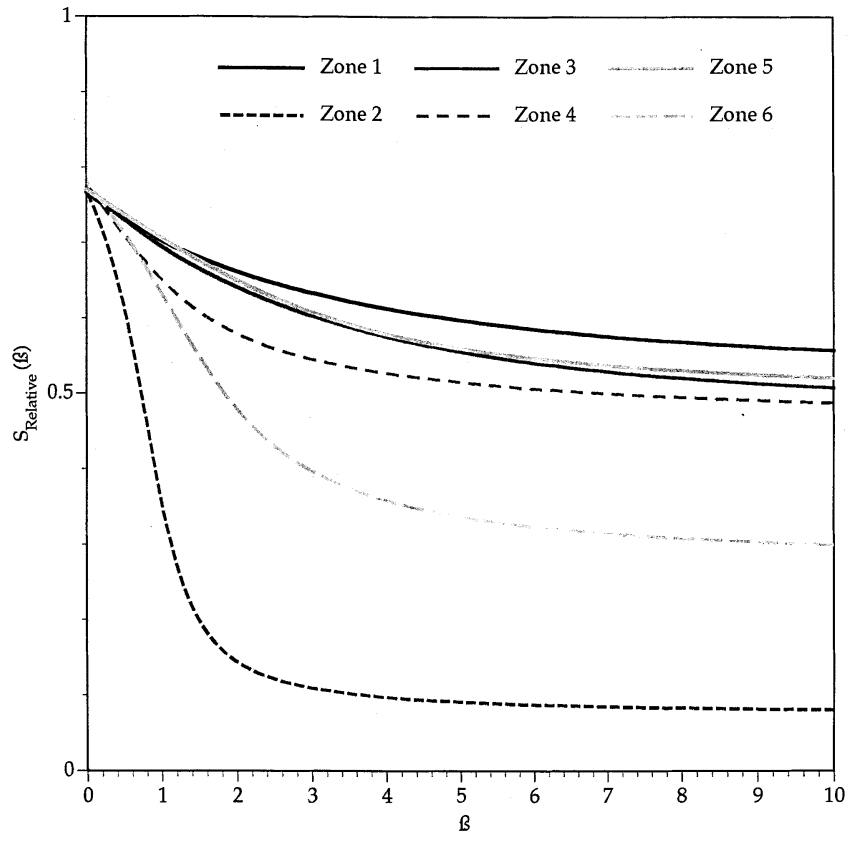

Figure 7. Relative Renyi entropies for $\beta=0$ to 10 after 3 pitches in the extruder for specific initial zones with 4,000 particles per zone and 2,880 bins.
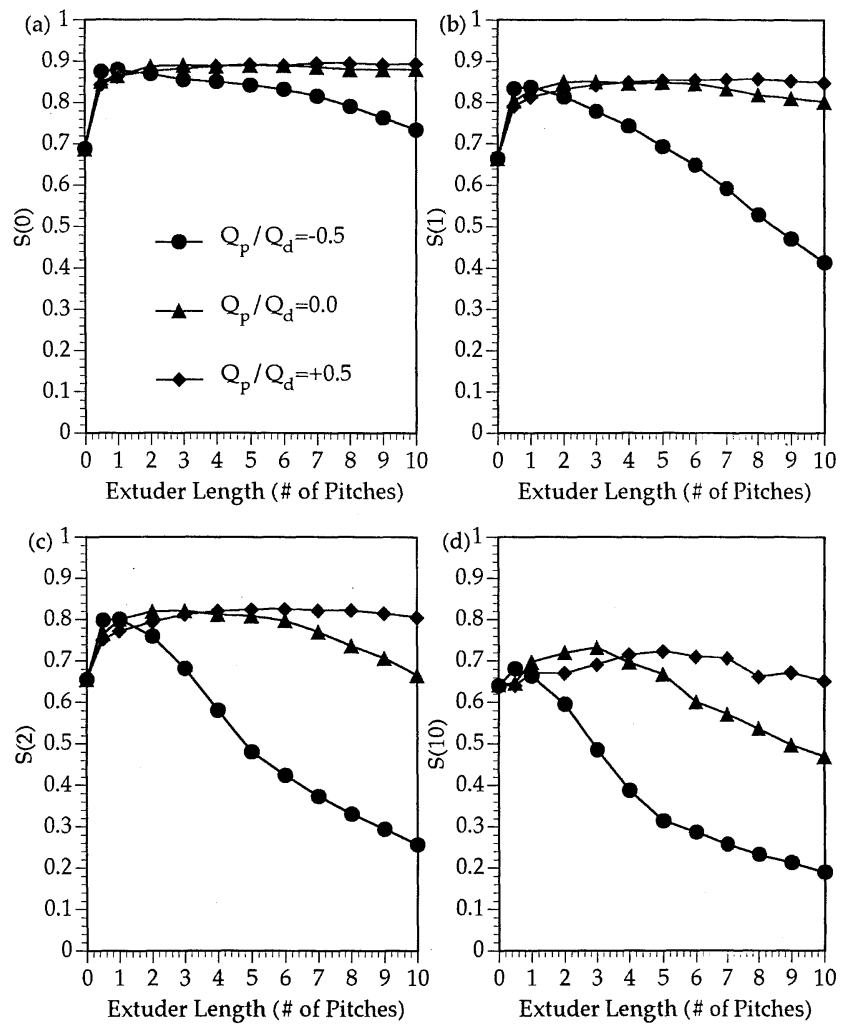

Figure 8. Evolution of the relative Renyi entropies for throttle ratio of $-0.5,0$ and +0.5 for (a) $\beta=0$, (b) $\beta=1$, (c) $\beta=2$, and (d) $\beta=10$. 

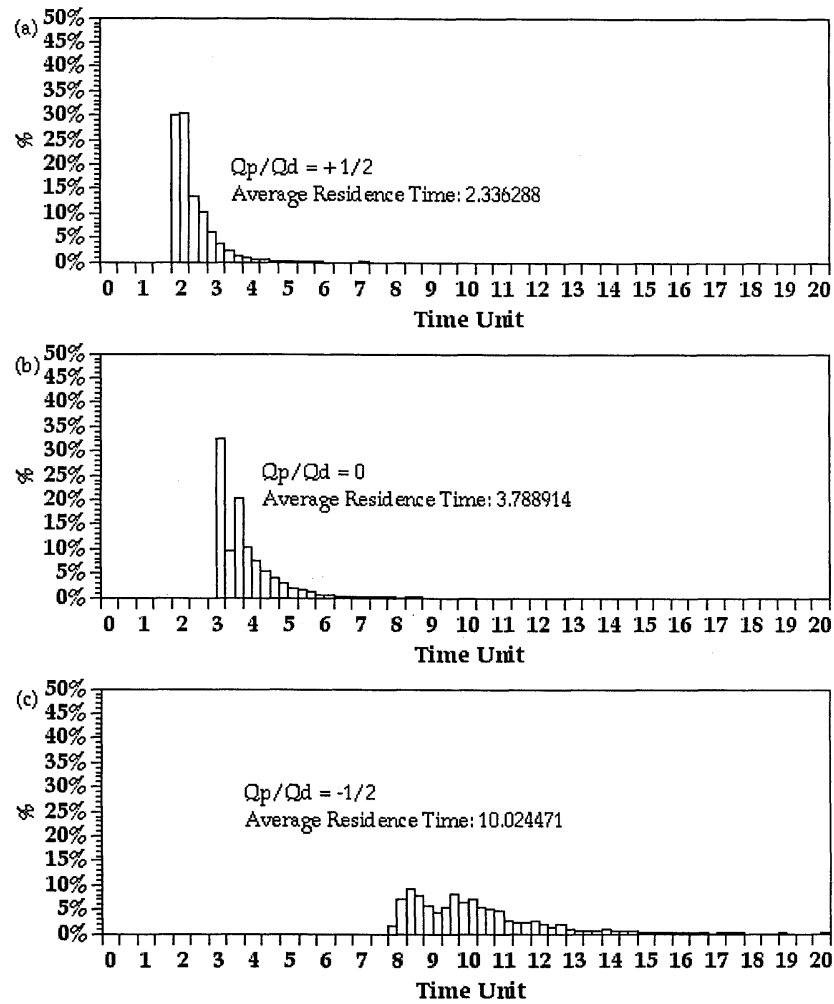

Figure 9. External residence time distributions for two pitches of the extruder for throttle ratios of (a) +0.5 , (b) 0 , and (c) -0.5 .

The data for three zones from the same angular position, but different radial positions show lower Renyi entropies for particles closer to the barrel. The results in Figure 7 point out to a direct correlation between the residence time of particles in the extruder and the degree of mixing they achieve. We corroborate next this observation concerning the influence of residence time on the quality of mixing. One simple way of changing the residence time in the equipment is through changing the throttle ratio $Q p / Q d$. Renyi entropies for three different operating conditions are shown in Figure 8. It is evident that, for negative $Q p / Q d$ values, the peak in the Renyi entropies, thus better distributive mixing, occurs in shorter extruders. An examination of Figure 9 illustrating the external residence time distributions for the three conditions explains why that is the case. As the pressure flow opposes the drag flow (negative $Q p / Q d$ ratio), the particles spend more time in each pitch, resulting in better mixing with shorter extruders. The average residence time in a two-pitch extruder is 4.3 times longer for the condition of $Q p / Q d=-0.5$ than for $Q p / Q d=+0.5$.

\section{Conclusions}

We have used Renyi entropies to characterize distributive mixing in continuous polymer processing equipment. Distributive mixing characterization based on Renyi entropies requires less computational time than the traditional method based on correlation dimension from correlation sums, and produces similar results. Renyi entropies are dependent on a $\beta$ parameter, which represents weighting on the concentration of the minor component. We have shown how, using different values of the $\beta$ parameter, different aspects of mixing quality can be assessed. Renyi entropies offer the advantage of a generalized form that can be tailored to the specific need, including focusing on void volumes $(\beta=0)$, and areas of highest concentration of minor component $(\beta \rightarrow \alpha)$. Depending on the requirements of the final product, design and operating specifications can be adjusted to maximize the particular Renyi entropy. While not yet experimentally verified, it appears from our numerical results that this particular design of twin flight single screw melt extruders offers no improvement in distributive mixing at lengths exceeding 6 pitches when operated at a positive throttle ratio of 0.5. By changing the throttle ratio, the optimal extruder length varied. A positive correlation was found between the optimal length and the average residence time.

\section{Acknowledgments}

The authors acknowledge the financial support of this work by the National Science Foundation through grants DMI-9812969 and DMI-0140412. We would also like to acknowledge the use of computing services from Ohio Supercomputer Center.

\section{Literature Cited}

Allen, P. A., M. Kaufman, A. F. Smith, and R. E. Propper, "A Molar Entropy Model of Age Differences in Spatial Memory," Psychology \& Aging, 13, 501 (1998).

Anderson, P. D., O. S. Galaktionov, G. W. M. Peters, F. N. Van de Vosse, and H. E. H. Meijer, "Analysis of Mixing in Three-Dimensional Time-Periodic Cavity Flows," J. Fluid Mech., 386, 149 (1999).

Avalosse, T., "Numerical Simulation of Distributive Mixing in 3-D Flows," Macromol. Symp., 112, 91 (1996).

Brooks, D. R., Evolution as Entropy, University of Chicago Press, Chicago (1988).

Bunde, A., S. Havlin, and H. E. Roman, "Multifractal Features of Random Walks on Random Fractals," Phys. Rev. A, 42, 6274 (1990).

Chakravarthy, V. S., and J. M. Ottino, "Mixing of Two Viscous Fluids in a Rectangular Cavity," Chem. Eng. Sci., 51, 3613 (1996).

Cheng, H., and I. Manas-Zloczower, "Chaotic Features of Flow in Polymer Processing Equipment-Relevance to Distributive Mixing," Int. Poly. Process., 12, 83 (1997).

Cheng, H., and I. Manas-Zloczower, "Distributive Mixing in Conveying Elements of a ZSK-53 Co-rotating Twin Screw Extruder," Poly. Eng. Sci., 38(6), 926 (1998).

Danckwerts, P. V., "The Definition and Measurements of Some Characteristics of Mixtures," Appl. Sci. Res., 279 (1953).

Galaktionov, O. S., P. D. Anderson, P. G. M. Kruijt, G. W. M. Peters, and H. E. H. Meijer, "A Mapping Approach for Three-Dimensional Distributive Mixing Analysis," Comput. Fluids, 30(3), 271 (2001).

Gao, J., G. C. Walsh, D. Bigio, R. M. Briber, and M. D. Wetzel, "Mean Residence Time Analysis for Twin Screw Extruders," Poly. Eng. Sci., 40(1), 227 (2000).

Gasner, G. E., D. Bigio, C. Marks, F. Magnus, and C. Kiehl, “A New Approach to Analyzing Residence Time and Mixing in a Co-rotating Twin Screw Extruder," Poly. Eng. Sci., 39, 286 (1999).

Georgescu-Roegen, N., The Entropy Law and the Economic Process, Harvard University Press, Cambridge, MA, p. 457 (1987).

Grassberger, P., and I. Procaccia, "Characterization of Strange Attractors," Phys. Rev. Lett., 50, 346 (1983).

Grassberger, P., and I. Procaccia, "Dimensions and Entropies of Strange Attractors From a Fluctuating Dynamics Approach," Physica $D, \mathbf{1 3}, 34$ (1984).

Ivanov, P. C., L. A. N. Amaral, A. L. Goldberger, S. Havlin, M. G. Rosenblum, Z. R. Struzik, and H. E. Stanley, "Multifractality in Human Heartbeat Dynamics," Nature, 399, 461 (1999). 
Kantz, H., and T. Schreiber, "Dimension Estimates and Physiological Data," Chaos, 5(1), 143 (1995).

Kurths, J., A. Voss, P. Saparin, A. Witt, H. J. Kleiner, and N. Wessel, "Quantative Analysis of Heart Rate Variability," Chaos, 5(1), 88 (1995).

Kwon, T. H., J. W. Joo, and S. J. Kim, “Kinematics and Deformation Characteristics As a Mixing Measure in the Screw Extrusion Process," Poly. Eng. Sci., 34, 174 (1994).

Lawal, A., and D. M. Kalyon, "Mechanisms of Mixing in Single and Co-Rotating Twin-Screw Extruders,” Poly. Eng. Sci., 35, 1325 (1995).

Lee, T. H., and T. H. Kwon, "A New Representative Measure of Chaotic Mixing in a Chaos Single-Screw Extruder," Adv. Poly. Technol., 18, 53 (1999).

Li, T., and I. Manas-Zloczower, "Evaluation of Distributive Mixing Efficiency in Mixing Equipment," Chem. Eng. Commun., 139, 223 (1995a).

Li, T., and I. Manas-Zloczower, "A Study of Distributive Mixing in Counterrotating Twin Screw Extruders," Int. Poly. Process., 10, 314 (1995b).

Mackley, M. R., and R. Saraiva, "The Quantitative Description of Fluid Mixing Using Lagrangian- and Concentration-Based Numerical Approaches," Chem. Eng. Sci., 54, 159 (1999).

Ottino, J. M., The Kinematics of Mixing: Stretching, Chaos, and Transport, Cambridge University Press, Cambridge (1989).
Renyi, A., Probability Theory, North-Holland Series in Applied Mathematics and Mechanics, Vol. 10, North-Holland, Amsterdam, p. 666 (1973).

Shannon, C. E., "The Mathematical Theory of Communication," Bell System Tech. J., 27, 379 (1948).

Shearer, G., and C. Tzoganakis, "Analysis of Mixing During MeltMelt Blending in Twin Screw Extruders Using Reactive Polymer Tracers," Poly. Eng. Sci., 39, 1584 (1999).

Wang, W., and I. Manas-Zloczower, "Temporal Distributions: A New Tool for the Development of Mixing Indexes for Scale-up of Polymer Processing Equipment," Poly. Eng. Sci., 41, 1068 (2001).

Wang, W., I. Manas-Zloczower, and M. Kaufman, "Characterization of Distributive Mixing in Polymer Processing Equipment Using Renyi Entropies,' Int. Poly. Process., 16, 315 (2001).

Wong, T. H., and I. Manas-Zloczower, "2-Dimensional Dynamic Study of the Distributive Mixing in an Internal Mixer," Int. Poly. Process., 9, 3 (1994).

Yao, W. G., K. Takahashi, and Y. Abe, "Analytical Study on Flow and Distributive Mixing of a New Type Pin Mixing Section for Screw Extruder," Int. Poly. Process., 11, 222 (1996).

Yoshinaga, M., S. Katsuki, M. Miyazaki, L. Liu, S.-I. Kihara, and K. Funatsu, "Mixing Mechanism of Three-Tip Kneading Block in Twin Screw Extruders," Poly. Eng. Sci., 40, 168 (2000).

Manuscript received Apr. 26, 2002, and revision received Feb. 14, 2003.

Post-print standardized by MSL Academic Endeavors, the imprint of the Michael Schwartz Library at Cleveland State University, 2017. 